\title{
Image Compression of MRI Image using Planar Coding
}

\author{
Lalitha Y. S \\ Department of Electronics \& Communication Engg. \\ Appa Institute of Engineering \& Technology
}

\begin{abstract}
In this paper a hierarchical coding technique for variable bit rate service is developed using embedded zero block coding approach. The suggested approach enhances the variable rate coding by zero tree based block-coding architecture with Context Modeling for low complexity and high performance. The proposed algorithm utilizes the significance state-table forming the context modeling to control the coding passes with low memory requirement and low implementation complexity with the nearly same performance as compared to the existing coding techniques.
\end{abstract}

Keyword- image coding; embedded block coding; context modeling; multi rate services.

\section{INTRODUCTION}

With rapid development of heterogeneous services in image application the future digital medical images and video coding applications finds various limitations with available resource. The traditional multi-bit stream approach to the heterogeneity issue is very constrained and inefficient under multi bit rate applications. The multi bit stream coding techniques allow partial decoding at a various resolution and quality levels. Several scalable coding algorithms have been proposed in the international standards over the past decade, but these former methods can only accommodate relatively limited decoding properties. The rapid growth of digital imaging technology in conjunction with the ever-expanding array of access technologies has led to a new set of requirements for image compression algorithms. Not only are high quality reconstructed medical images required at medium-low bitrates, but also as the bit rate decreases, the quality of the reconstructed MRI image should degrade gracefully. The traditional multi-bit stream solution to the issue of widely varying user resources is both inefficient and rapidly becoming impractical. The bit level scalable codes developed for this system allow optimum reconstruction of a medical image from an arbitrary truncation point within a single bit stream. For progressive transmission, image browsing, medical image analysis, multimedia applications, and compatible trans coding, in a digital hierarchy of multiple bit rates, the problem of obtaining the best MRI image quality and accomplishing it in an embedded fashion i.e. all the encoded bits making compatible to the target bit rate is a bottleneck task for today's engineer. As medical images are of huge data set and encoding it for a lower bit rate results in loss of data, which intern results in very low image quality under compression. Coming to the transmission over a noisy channel this problem becomes more effective due to narrow bandwidth effect. Various algorithms were proposed for encoding and compressing the MRI image data before transmission. These algorithms show high-end

\author{
Mrityunjaya V. Latte \\ Principal, JSS Academy of Technical Education, \\ Bangalore, Karnataka, India Gulbarga, Karnataka, India.
}

results under high bandwidth systems but show poor result under low data rate systems. The problem of transmission of MRI images over a low bit rate bandwidth can be overcome if the medical image data bits are such encoded and compressed that the data bit rate is made compatible to the provided low bit rate. Embedded zero tree wavelet algorithm is a proposed image compression algorithm which encode the bit in the bit stream in the order of importance which embed the bit stream in hierarchical fashion.

\section{System Design}

This work was motivated by success of two popular embedded coding techniques: zero-tree/-block coding [1, 2, 3, 4] and context modeling of the sub band/wavelet coefficients $[5,6,7]$. Zero-tree/-block coding takes advantage of the nature of energy clustering of sub band/wavelet coefficients in frequency and in space. These classes of coders apply a hierarchical set partitioning process to split off significant coefficients (with respect to the threshold in the current bit plane coding pass), while maintaining areas of insignificant coefficients. In this way, a large region of zero pixels can be coded into one symbol. It provides an efficient method to compactly represent a group of leading zeros of sub band/wavelet coefficients. The distinguished compression performances were demonstrated in [2, 3, 4]. Moreover, instead of all pixels, only a small number of elements in lists [2] needs to be processed in individual bit plane coding passes. Thus, processing speed for this class of coders is very fast. High compression efficiency achieved with context modeling was presented in $[5,6,7]$. In this class of coders, individual pixel of the DWT bit planes are coded using context based arithmetic coding. With help of the context models, strong correlation of sub band/wavelet coefficients within and across sub bands can be effectively utilized. Although simple context modeling was also employed in [2, 3, 4], the limited context information in those algorithm were insufficient to accurately predict the status of the current node. With carefully designed context models, some algorithms $[6,7]$ have been able to outperform the best zero-tree/block coders in PSNR performances. Nevertheless, unlike zero-tree/-block coders, these algorithms needed to scan all sub band/wavelet coefficients at least once to finish coding of a full bit plane, with an implied higher computation cost. To combine advantages of these two coding techniques, ie, low computation complexity and effective exploitation of correlation of sub band coefficients, we propose an embedded medical image coding algorithm using Zero Blocks of sub band/ wavelet coefficients and context modeling, or EZBC for ease of reference. This zero block coding 
algorithm is also based on the set partitioning technique. We adopted the adaptive quad tree splitting method introduced in [3] to separate the significant coefficients and code every block of zero pixels into one symbol. In this scheme, quad tree representations of DWT coefficients are first established for individual sub bands. The bottom level of the quad tree consists of the sub band/wavelet coefficients. The single node at the top tree level, or the root node, just corresponds to the maximum amplitude of the all DWT coefficients. To start with, the root is the only insignificant node to process. Each quad tree node splits into four insignificant descendent nodes of the next lower level once it tests as significant against the threshold of the current bit plane coding pass. The same splitting process is recursively applied to the individual descendent nodes until the bottom level of the quad tree is reached.

In this way, we can quickly zoom in to high-energy areas and regions of all zero pixels can be compactly represented. In EZBC, the context models were carefully designed for coding quad tree nodes at different tree levels and sub bands. Therefore, it retains the properties of compactness and low complexity of the zero block coders, and adds context information in an effective way, while the context information is also made effective use. Unlike the zero tree structure, each zero block only represents pixels from one sub band. Hence, EZBC is inherently applicable to resolution scalable applications. With the aid of inter band context, dependence of sub band/wavelet coefficients across scales can still be effectively utilized without having zero trees spanning several sub bands.

\section{Medical IMAge Coding System}

Image compression addresses the problem of reducing the amount of data required to represent a digital medical image. Compression is achieved by the removal of one or more of three basic data redundancies: (1) coding redundancy, which is present when less than optimal (i.e., the smallest length) code words are used; (2) inter pixel redundancy, which results from correlations between the pixels of an medical image; and/or (3) psycho visual redundancy, which is due to data that is ignored by the human visual system (i.e., visually nonessential information).In this chapter We examine each of these redundancies, describe a few of the many techniques that can be used to exploit them, and examine two important compression standards - JPEG and JPEG 2000. These standards unify the concepts by combining techniques that collectively attack all three data redundancies. Medical Image compression systems are composed of two distinct structural blocks: an encoder and a decoder. Image $f(x, y)$ is fed into the encoder, which creates a set of symbols from the input data and uses them to represent the medical image. If we let $n_{1}$ and $n_{2}$ denote the number of information carrying units (usually bits) in the original and encoded medical images, respectively, the compression that is achieved can be quantified numerically via the compression ratio

$$
C_{R}=\frac{n_{1}}{n_{2}}
$$

To view and/or use a compressed (i.e., encoded) medical image, it must be fed into a decoder (see Fig.2.1), where a reconstructed output medical image, $\hat{f}(x, y)$, is generated. In general, $\hat{f}(x, y)$ may or may not be an exact representation of $f(x, y)$. If it is, the system is called error free, information preserving, or lossless; if not, some level of distortion is present in the reconstructed medical image. In the latter case, which is called lossy compression, we can define the error $e(x, y)$ between $f(x, y)$ and $\hat{f}(x, y)$, for any value of $x$ and $y$ as

$$
e(x, y)=\hat{f}(x, y)-f(x, y)
$$

so that the total error between the two medical images is

$$
\sum_{x=0}^{M-1} \sum_{y=0}^{N-1}[y(x, y)-f(x, y)]
$$

and the rms (root-means-square) error $e_{r m s}$ between $f(x, y)$ and $\hat{f}(x, y)$ is the square root of the squared error averaged over the $M \times N$ array, or

$$
e_{r m s}=\left[\frac{1}{M N} \sum_{x=0}^{M-1} \sum_{y=0}^{N-1}[y(x, y)-f(x, y)]^{2}\right]^{1 / 2}
$$

In the first stage of the encoding process, the mapper transforms the MRI input image into a (usually nonvisual) format designed to reduce inter pixel redundancies. The second stage, or quantizer block, reduces the accuracy of the mapper's output in accordance with a predefined fidelity Criterionattempting to eliminate only psycho visually redundant data. This operation is irreversible and must be omitted when errorfree compression is desired. In the third and final stage of the process, a symbol coder creates a code (that reduces coding redundancy) for the quantizer output and maps the output in accordance with the code.

\section{Bit Plane Coding}

Wavelet coefficient bit plane coding in EZBC follows a similar procedure to those adopted in other quad tree-based set partitioning coders. However, special care is given to list management and bit stream organization, which significantly influence efficiency of context modeling and code stream embedding, and scalable functionality of the resulting code stream. The EZW encoder is based on two important observations:

1) Natural MRI images in general have a low pass spectrum. When a medical image is wavelet transformed the energy in the sub bands decreases as the scale decreases (low scale means high resolution), so the wavelet coefficients will, on average, be smaller in the higher sub bands than in the lower sub bands. This shows that progressive encoding is a very natural choice for compressing wavelet transformed medical images, since the higher sub bands only add detail.

2) Large wavelet coefficients are more important than small wavelet coefficients. 
These two observations are exploited by encoding the wavelet coefficients in decreasing order, in several passes. For every pass a threshold is chosen against which all the wavelet coefficients are measured. If a wavelet coefficient is larger than the threshold it is encoded and removed from the image, if it is smaller it is left for the next pass. When all the wavelet coefficients have been visited the threshold is lowered and the MRI image is scanned again to add more detail to the already encoded MRI image. This process is repeated until all the wavelet coefficients have been encoded completely or another criterion has been satisfied (maximum bit rate for instance).

The trick is now to use the dependency between the wavelet coefficients across different scales to efficiently encode large parts of the MRI images which are below the current threshold. It is here where the zero tree enters. So, let me now add some detail to the foregoing. (As most explanations, this explanation is a progressive one.)

The EZW encoder exploits the zero tree based on the observation that wavelet coefficients decrease with scale. It assumes that there will be a very high probability that all the coefficients in a quad tree will be smaller than a certain threshold if the root is smaller than this threshold. If this is the case then the whole tree can be coded with a single zero tree symbol. Now if the MRI image is scanned in a predefined order, going from high scale to low, implicitly many positions are coded through the use of zero tree symbols. Of course the zero tree rule will be violated often, but as it turns out in practice, the probability is still very high in general. The price to pay is the addition of the zero tree symbol to our code alphabet.

Quad tree Structure The bit plane coding process begins with establishment of the quad tree representations for the individual sub bands. The value of a quad tree (a) Quadtree buildup (b) Quadtree splitting node $Q_{k}[l](i, j)$ at position (i, $\mathrm{j})$, quad tree level $l$ and subband $\mathrm{k}$ is defined by $Q_{k}[0](i, j) \square\left|c_{k}(i, j)\right|$, and

$$
\begin{gathered}
Q_{k}[l](i, j) \square \max \left\{Q_{k}[l-1](2 i, 2 j), Q_{k}[l-1](2 i, 2 j+1),\right. \\
\left.Q_{k}[l-1](2 i+1,2 j), Q_{k}[l-1](2 i+1,2 j+1)\right\},
\end{gathered}
$$

Where $c_{k}(i, j)$ is the subband coefficient at position $(\mathrm{i}, \mathrm{j})$, subband $\mathrm{k}$. That is, each bottom quadtree node is assigned to the magnitude of the subband coefficient at the same position. The quadtree node at the next higher level is then set to the maximum of the four corresponding nodes at the current level, as illustrated in Fig. 1 (a). By recursively grouping each $2 \times 2$ vector this way, the complete quad tree is built up for the individual subbands. The top quad tree node $Q_{k}\left[D_{k}-1\right](0,0)$ is just equal to the maximal magnitude of all subband coefficients $\left\{c_{k}(i, j)\right\}_{\mathrm{i}, \mathrm{j}}$ from subband $\mathrm{k}$, where $\mathrm{D}_{\mathrm{k}}$ is the quadtree depth for subband $\mathrm{k}$.
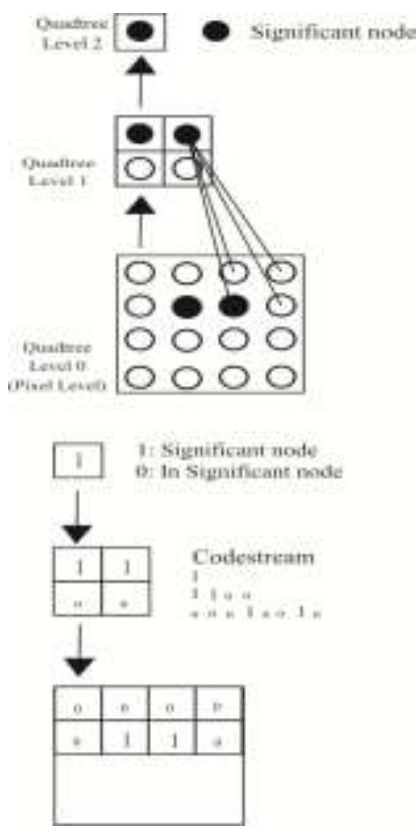

Fig. 1 Illustration of quad tree build up and decomposition.

Similar to the conventional bitplane coders, we progressively encode subband coefficients from the MSB toward the LSB. The individual bitplane coding pass $\mathrm{n}$ encodes bit $\mathrm{n}$ of all coefficients. The subband coefficients are thus effectively quantized by a family of embedded quantizers with a (dead-zone) quantization threshold $\tau_{\mathrm{n}}=2^{\mathrm{n}}$ for bitplane level $\mathrm{n}$. We define that a quadtree node $\mathrm{Q}$ tests significant with respect to a quantization threshold $\tau$ if $\mathrm{Q} \geq \tau$. A quadtree $\mathrm{Q}$ is defined to be a significant node during the current bitplane pass $\mathrm{n}$ if

$$
\begin{gathered}
-Q \geq \tau_{n+1} \text {, or } \\
-Q \in\left[\tau_{n}, \tau_{n+1}\right) \text { and } \mathrm{Q}
\end{gathered}
$$

has been tested, and an insignificant node otherwise. It should be noted that $Q \in\left[\tau_{n}, \tau_{n+1}\right)$ is still considered as an insignificant node in the bitplane pass $\mathrm{n}$ until it has been coded/tested. A significant pixel (coefficient) is located by the testing and splitting operation recursively performed on the significant nodes up to the pixel (bottom) level of a quadtree, as shown in Fig. 1 (b). Given the coded quadtree splitting decisions, the decoder can duplicate the quadtree decomposition steps and the related significance information.

A parent-child relationship is defined in the quadtree structure. As opposed to the classic zerotree structure, this relationship is between nodes across the quadtree levels (rather than across resolution scales), as illustrated in Fig. 3.5. Each parent $Q_{k}[l](i, j)$ has four children from the same $2 \times 2$ block at the next lower quadtree level,. These four child nodes are considered as siblings of each other. All the descendants of $Q_{k}[l](i, j)$ can be recursively traced from one quadtree level to the next lower in a similar way up to the pixel level.

Every quadtree node Q plays a dual role: It is an element with the value defined by (3.1) subjected to significance test. The established quadtree representation provides an additional 
pyramidal description of the transformed medical image. The strong statistical dependencies among quadtree nodes, can be exploited to improve the performance of conditional entropy coding. Once a node Q tested insignificant, it indicates that all its descendants are insignificant, too. Hence, it also serves as a zero set defined in the conventional set partitioning coder and contains all its descendent coefficients as members. Each insignificant quadtree node $\mathrm{Q}[l](\mathrm{i}, \mathrm{j})$ thus effectively groups $2^{l} \times 2^{l}$ insignificant coefficients together. The hierarchical set partitioning with respect to a given test threshold is accomplished via recursive quadtree splitting.

Similar to other hierarchical coders, we utilize the ordered lists for tracking the significance status of the individual subband coefficient. $\mathrm{LSP}_{\mathrm{k}}$ (list of significant pixels) contains a full list of significant pixels in subband $\mathrm{k}$. All the insignificant coefficients are compactly gathered in the quadtree nodes which are maintained in arrays of the LINs (list of insignificant nodes). $\operatorname{LIN}_{\mathrm{k}}[l], l=0, \ldots, D_{k}-1$, contains a list of insignificant nodes from quadtree level 1 , subband $\mathrm{k}$. A node $(\mathrm{i}, \mathrm{j})$ in $\operatorname{LIN}_{\mathrm{k}}[l]$ thus indicates that $2^{L} \times 2^{L}$ subband coefficients

$$
\begin{gathered}
\left\{\left\{c_{k}\left(i \cdot 2^{l}, j \cdot 2^{l}\right), c_{k}\left(i \cdot 2^{l}, j \cdot 2^{l}+1\right), \ldots,\right.\right. \\
\left.c_{k}\left(i \cdot 2^{l}, j \cdot 2^{l}+2^{l}-1\right)\right\},\left\{c_{k}\left(i \cdot 2^{l}+1, j \cdot 2^{l}\right), \ldots,\right. \\
\left.c_{k}\left(i \cdot 2^{l}+1, j \cdot 2^{l}+2^{l}-1\right)\right\}, \ldots, c_{k}\left(i \cdot 2^{l}+2^{l}-1, j \cdot 2^{l}\right), \ldots, \\
\left.\left.c_{k}\left(i \cdot 2^{l}+2^{l}-1, j \cdot 2^{l}+2^{l}-1\right)\right\}\right\}
\end{gathered}
$$

are all insignificant. Unlike other earlier set partitioning coders such as EZW, SPIHT and SPECK, the lists here are maintained separately for the individual subbands and quadtree levels. This strategy can effectively improve performance of context modeling and codestream embedding. Moreover, it is essential for efficient accommodation of resolution-scalable codestreams.

Bitplane Processing and Coding Initially, $\operatorname{LIN}_{\mathrm{k}}\left[\mathrm{D}_{\mathrm{k}}-1\right]$ contains the single node from the top quadtree level and the other $\mathrm{LIN}_{\mathrm{k}}$ lists and $\mathrm{LSP}_{\mathrm{k}}$ are just empty for each subband $\mathrm{k}$. It indicates that all the subband coefficients are insignificant before the first bitplane coding pass starts. The coding process begins with the MSB plane with the bit index given by

$$
n_{\max } \square\left\lfloor\log _{2}\left(\max _{(k, i, j)}\left\{\left|c_{k}(i, j)\right|\right\}\right)\right\rfloor
$$

where $\lfloor\cdot\rfloor$ returns the largest integral value not greater than the input.

Because the quantization threshold is halved from bitplane to bitplane, more and more coefficients becomes significant as the bitplane coding process proceeds.

Two tasks are performed in each bitplane pass n:

1) 1. Reveal the new significant coefficients, $\{c|| c \mid \in[$ $\left.\left.2^{n}, 2^{n+1}\right)\right\}$ with respect to the current threshold $\tau_{n}=2^{n}$;

2) 2. Refine the old significant coefficients, $\left\{c|| c \mid \geq 2^{n+1}\right\}$, collected from the previous bitplane passes.
Since all the insignificant coefficients with respect to the previous test threshold $\tau_{n}=2^{n+1}$ were already compactly grouped by the quadtree nodes maintained in the LINs, the new significant coefficients can be fast located by significance test of the individual nodes from the LINs. If a node is tested insignificant, it remains in the same LIN. Otherwise, it splits into four children and each child is further tested. Once a child is tested insignificant, it is added to the LIN at the next lower level. Otherwise, it further splits into four grandchildren and each is tested in a similar way. This testing and splitting procedure is recursively performed on the significant descendants up to the pixel level. As soon as a pixel tested significant, it is appended to the LSP and its sign is encoded immediately. The refinement of old significant coefficients (socalled refinement pass) is simply accomplished by coding bit $\mathrm{n}$ of each coefficient in the LSPs gathered from the previous bitplane passes (not including the new significant coefficients from the current pass). The Boolean results of significance tests (true or false), the sign bits, and the refinement bits are all the required information for decoding of bitplane data. They are all encoded by context-dependent binary arithmetic coding, to be presented shortly. The bitplane coding process will stop once the desired coding bitrate or image quality is reached.

The quadtree build-up stage is not needed at the decoder as the significance information is already contained in the codestream. The bitplane decoder basically follows the same procedure as the bitplane encoder. Given the coded sequences of quadtree splitting decisions, the decoder can duplicate the quadtree decomposition steps taken by the encoder. The execution path within a subband for bitplane encoding is required to be strictly followed for bitplane decoding. However, since the quadtrees are independently established for the individual subbands, the bitplane decoding order among subbands is allow to be manipulated in various ways for some scalable coding applications.

In addition to the subband coefficients, the proposed algorithm also needs to deal with the quadtree nodes from the individual quadtree levels. At first look, it appears that the data size for processing and coding in each bitplane pass has been significantly expanded. Nevertheless, none of these quadtree nodes will be visited until their parents is already tested significant, except for the top quadtree nodes which do not have any parents. On the contrary, our experimental results show that the number of coded binary symbols in EZBC is in fact even smaller than that of pixels in the input medical image (at a typical lossy coding bitrate). That is, the average number of binary coding operation per pixel is less than one although the individual subband coefficient is encoded through multiple quantization and coding stages. It partially explains the high speed performance of this block-based set partitioning coding scheme.

Still, we need to visit every coefficient once for establishment of the quadtree representations of the decomposed medical image at the very beginning of the bitplane coding process. Nevertheless, our coding algorithm in fact is only concerned with the MSB index of a quadtree node (rather than the actual value) for significance test. This 
quadtree buildup step can thus be efficiently implemented by the simple bitwise OR operation.

In order to have efficiently embedded codestreams, it is essential that the code data in the compressed file are ordered according to their relative efficiencies for distortion reduction. This basic concept is commonly called embedding principle. In the proposed algorithm, a fixed path for encoding of wavelet coefficient bitplane data is chosen as follows: The coding process advances in a bitplane-wise fashion from the most significant bit toward the least. In a given bitplane, the arrays of LINs are processed in an increasing order of quadtree level, as suggested by Islam and Pearlman in SPECK. That is, all the pixels in LIN[0] are processed first and all the nodes in LIN[1] are then processed next, followed by the processing's of LIN[2], LIN[3], and so on. In this way, the busy areas in the transformed medical image are updated earlier via a few quadtree splitting and coding steps, resulting in a good ratedistortion performance. The refinement of the previous significant coefficients from LSP is executed at last. In a significance test pass of a given quadtree level or a coefficient refinement pass, the subbands are visited from coarse to high resolution

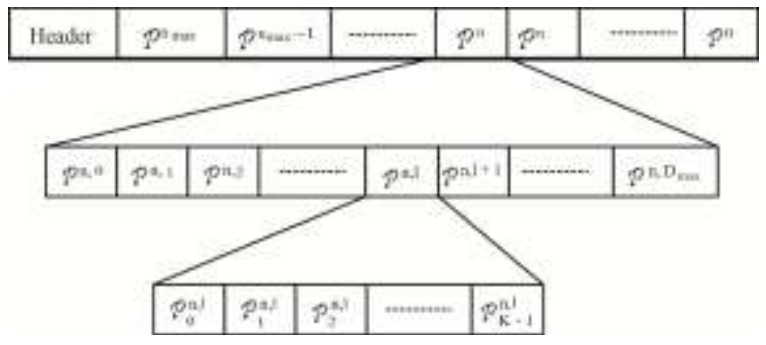

Fig. 2 Illustration of the hierarchical layout of a EZBC codestream.

A hierarchical layout of a EZBC codestream is depicted in Fig. 2, where $\mathrm{p}^{\mathrm{n}}$ denotes the bitplane pass $\mathrm{n}, p_{k}^{n, l}$ the subbitplane pass for processing the insignificant nodes in $\operatorname{LIN}_{k}[1]$ (routine CodeLIN $(\mathrm{k}, 1)$ ), and $p_{k}^{n, D_{\max }}$ the sub-bitplane pass for the refinement of the significant coefficients in $\mathrm{LSP}_{k}$ (routine CodeLSP $(\mathrm{k})$ ). Similar to the bitplane de-interleaving scheme widely adopted in the sequential bitplane coders, EZBC effectively partitions each bitplane into multiple sub-bitplane passes $\left\{p_{k}^{n, l}\right\}_{\mathrm{n}, \mathrm{l}, \mathrm{k}}$ for providing an embedded codestream of fine granularity. However, unlike the multi-pass approach proposed in, EZBC does not need to scan the individual pixels more than once in each bitplane pass because all the involved pixels for the individual sub-pass were already organized in separate lists. Although our pre-defined data embedding order is not optimized for the best R-D performance (as compared to the algorithms), our empirical data show the resulting relative performance loss is mostly insignificant. The effectiveness of the proposed data embedding strategy is further evidenced by the smooth R-D curves shown in our actual coding simulation results

It is worth mentioning that each bitplane pass could have been divided into even more sub-bitplane passes in our data embedding scheme to further improve the R-D performance of the resulting codestream. It is simply accomplished by partitioning of the existing lists into smaller sub-lists and then processing each sub-lists via separate sub-bitplane coding passes. The resulting computational and storage costs are still the same because the total number of the nodes to be stored and processed in all the maintained lists is unchanged. For example, our empirical data show that the refinement of the significant coefficients from the previous bitplane coding pass reduces distortion more efficiently than the refinement of the significant coefficients from the other earlier bitplane coding passes (if exist). The PSNR performance can thus be slightly improved by partitioning the existing refinement pass into multiple subpasses, each for the refinement of the significant coefficients from particular bitplane level(s). Nevertheless, it is observed that the granularity of the resulting codestream by the current algorithm is already fine enough in practical medical image coding applications.

\section{A. Context-dependent entropy coding}

As opposed to the conventional sequential bitplane coder, the proposed algorithm EZBC is required to process the bitplanes associated with the individual quadtree levels in each bitplane coding pass. A dual hierarchical pyramidal description, as previously shown in Fig. 3.1, is thus given by this quadtree representation of the decomposed medical images. Strong intraband correlation is clearly exhibited among quadtree nodes. The self-similarity is demonstrated across both quadtree and resolution levels. Such diverse statistical dependencies are exploited by context-dependent arithmetic coding in EZBC. Unlike most former set-partition coders, the lists in EZBC are separately maintained for the individual subbands and quadtree levels. Therefore, the independent probability models are allowed to be built up for significance coding of the nodes from different subbands and quadtree levels. As such, the unique statistical characteristics of the source samples associated with different subband orientations, sub-sampling factors and amplitude distributions will not be mixed up in the accumulated probability models.

Four classes of binary symbols are encoded in EZBC:

1) Significance test of a given quadtree node from LIN (in routine CodeLIN),

2) Significance test of a child (in routine Code Descendants)

3) Sign bit of a newly significant coefficient, and

4) Refinement bit of a given coefficient from LSP.

These symbols are all encoded by context-based arithmetic coding with the distinct modeling strategies, to be respectively described in the following.

\section{B. Significance coding}

This subsection begins with a brief discussion that relates the proposed entropy coding algorithm to the APSG (alphabet partitioning and sample grouping) scheme. The utilization of inter- and intra-band correlation and dependence between subband levels in a subband-based quadtree representation is then respectively described, followed by a description of our context quantization/selection scheme. 
Significance coding in the proposed algorithm is conditioned on the significance map defined by

$$
\sigma(i, j)=\left\{\begin{array}{l}
1, \text { if node }(i, j) \text { is significant } \\
0, \text { otherwise }
\end{array}\right.
$$

Nevertheless, in contrast to the conventional sequential pixel-wise bitplane coding approach, significance coding of a quadtree node in EZBC jointly encodes a group of coefficients from a block region. The significance status of a contextual quadtree node compactly indicates local activity level in the corresponding block area. Hence, we can think of EZBC as a conditional block entropy coder that applies the APSG scheme on both source samples and modeling contexts. As such, block entropy coding and conditional entropy coding can be combined to exploit the strong subband dependency without suffering the complexity difficulties commonly associated with high-order source extension and context modeling. Although from a point of view of the conventional universal source coding, it was argued that there is no additional compression gain by blocking samples in conditional entropy coding. However, our block coding method here does not involve complex codebook design or any other computation-intensive procedures. On the contrary, the speed performance of the resulting coding system is substantially improved by compact grouping of subband coefficients. Besides, arithmetic coding of large areas of zero coefficients individually in conventional sequential bitplane coding is associated with a highly skew probability distribution, which is known to be penalized by a high learning cost.

\section{Contextual Region}

In Fig. 3 (a), we show the neighboring nodes included in the modeling contexts in our coder EZBC for significance coding. The eight spatial adjacent nodes from the same quadtree level are utilized to exploit intraband correlation. Such a contextual structure has been widely employed in many conventional bitplane coders, for significance coding of subband coefficients. The application of this model to significance coding of quadtree nodes is justified by the strong spatial dependency exhibited in the example MSB map in Fig. 3 (b).

To exploit the subband correlation across scales, we adopt the corresponding node from the next lower quadtree level (rather than from the current level) in the parent subband, indicated by ' $F$ ' in Fig. 3 (a). This choice is based on the fact that at a given quadtree level the related dimension in an input medical image for a quadtree node is doubled in the parent subband, as a result of sub-sampling operation in the subband transformation stage. The inter-band information is thus provided at the same spatial resolution by the parent subband, as demonstrated in Fig. 3(b).

\section{Inter-/Intra-Band Modeling}

The importance of the inter-band neighboring pixels lies in its ability to provide non-causal contextual information for conditional entropy coding. Such information is particularly valuable for efficient compression of the leading bitplanes since most spatial neighboring coefficients are still insignificant. However, the complexity of the algorithm may be increased by a use of the inter-band neighbors for some imaging applications. It has been argued that the intra-band context model is capable of effective exploitation of
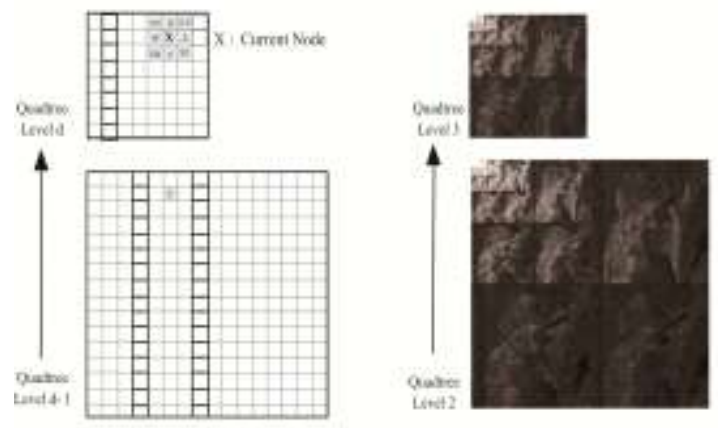

Fig. 3 Modeling contexts for conditional entropy coding of significance test. (a) Left: Neighbors included in the modeling contexts. (b) Right: Example MSB map of quadtree nodes from the decomposed MRI image.

Subband dependency combined with some efficient zero coding schemes. EBCOT, for instance, exhibits excellent compression performance combining quadtree decomposition, run-length coding with conventional context-based bitplane coding. Similar phenomenon was also observed during the development of EZBC. Our experimental results show that no PSNR improvement is made for significance coding of the individual pixels from LIN by inclusion of interband neighbors in the modeling context. The improvement for significance coding of a child pixel is also very limited. It is expected because the current pixel for significance test often already has some neighboring pixels tested significant when the quadtree decomposition operation proceeds to the bottom level. As a result, we only employ intraband modeling for conditional entropy coding of significance test at the pixel level.

On the other hand, it was also observed in our experimental results that the spatial dependency among quadtree nodes is decreasing as we move toward the higher quadtree levels. Recall that the value of a quadtree node is defined to be the maximal amplitude of all subband coefficients from its corresponding block region and the block size grows exponentially with the quadtree level. As is well known, the pixels with peak values are typically related to "singularity" in the input MRI image. Because of the energy clustering nature of subband coefficients, these medical image features are typically easily noticeable in the spatial contexts at the lower quadtree levels. Nevertheless, such clustering's of high energy are restricted to the local region around the "peak pixels". As the block size grows (or the quadtree level increases), this energy clustering phenomenon in the current block will become less likely reflected in the neighboring nodes if the peak pixel is not close to the block boundaries. Nevertheless, such an "anomaly" in space remains seen from the same corresponding area in the parent subband. Since the significance of a parent node is coded earlier during every bitplane pass, we can say that the parent node provides a look-ahead function into the region covered by the current node. In fact, our simulations indicate that the interband-only context model (containing a single neighboring node from the parent band) outperforms the 
intraband-only context model (containing eight spatial neighboring nodes) at the 3 rd level of the quadtree and higher.

The use of inter-band modeling is only optional in EZBC. An example application desirable for intraband modeling is highly scalable image coding presented.

\section{E. Dependency between Quadtree Levels}

The correlation between the adjacent quadtree levels is exploited to provide the inter-subband contextual information at the same spatial resolution. Within a given subband, the dependency also exists between the adjacent quadtree levels, directly attributed to the two properties associated with the basic mechanism of quadtree build-up and splitting:

1) When a parent remains insignificant, all its children are also insignificant;

2) After a parent tested significant, at least one of its four children will test significant in the subsequent descendant test

Property (i) is already utilized in a conventional zeroblock coder to compactly represent large numbers of insignificant pixels from block regions. Property (ii) implies that the chance of being testing significant is increasingly higher for the next child if none of its siblings have been tested significant yet. For example, without considering other contextual information, the probability that the first child tests significant is no less than 0.25 . Had none of the past three siblings been tested significant, the fourth child is significant for sure and no significance testing and coding is required. This statistical characteristic is exploited in our context modeling scheme detailed in the next section.

\section{F. Context Selection and Look-up tables}

Although adaptive arithmetic coding is a universal coding scheme which allows the source statistics to learn on the fly, the related learning costs often turn out quite expensive for many practical instances. The compression efficiency can be substantially improved if prior knowledge about the source is effectively exploited in the context model design.

Unlike the conventional sequential bitplane coder which encodes subband samples one by one in each bitplane coding pass, EZBC processes coefficients in groups and hence has fewer samples to encode. Context dilution thus becomes an issue of great concern. Our context modeling scheme is based on the significance map which allows context quantization to be implicitly carried out. Instead of treating all the resulting context vectors $\left(2^{9}\right.$ totally) as different conditional states, we carefully classify them into several model classes, similar to the context selection approach adopted in EBCOT and JPEG 2000. The look-up tables are then established accordingly to fast map a given context to the assigned model index. This strategy can further lower the model cost and enable the probability models to fast adapt themselves to varying local statistics.

The context classification, based upon the configurations of the significance map, is characterized by:

\section{1) Orientations: identified by}

$\mathrm{H}=\sigma(\mathrm{W})+\sigma(\mathrm{E})$, such that $0 \leq \mathrm{H} \leq 2$,

$\mathrm{V}=\sigma(\mathrm{N})+\sigma(\mathrm{S})$, such that $0 \leq \mathrm{V} \leq 2$,
$\mathrm{HV}=\mathrm{H}+\mathrm{V}$, such that $0 \leq \mathrm{HV} \leq 4$, and

$\mathrm{D}=\sigma(\mathrm{NW})+\sigma(\mathrm{NE})+\sigma(\mathrm{SW})+\sigma(\mathrm{SE})$, such that

$0 \leq \mathrm{D} \leq 4$

where the relative positions of nodes ' $\mathrm{W}$ ', 'E', 'N', 'S', 'NW', 'NE', 'SW',

'SW' are shown in Fig. 3.

2) Inter-band dependency: identified by

$P=\sigma(F)$, such that, $0 \leq p \leq 1$,

where the position of node ' $\mathrm{F}$ ' is shown in Fig. 3

3) Relative position $v$ : index of the current child node as shown in Fig. 3. where

$\mathrm{v} \in\{00,01,10,11\}$.

4) Significance of the past coded siblings:

$$
\sigma_{s b}(v) \underline{\Delta}\left\{\begin{array}{l}
\left\{1, \text { if } v>0 \text { and } \sum_{i=0}^{v-1}(i)>0\right. \\
0, \text { otherwise }
\end{array}\right.
$$

Where $v$ is the index of the current child node.

The spatial correlation among quadtree nodes is exploited by the eight first order neighbors, as depicted in Fig.3. The structure features are summarized in horizontal, vertical and diagonal directions, respectively. It is well known that the MRI image attributes such as edges and corners are retained along the direction of lowpass filtering after subband transform. Hence, our context modeling scheme emphasizes the directional characteristics in accordance with the current subband orientation. For instance, the horizontal features are favored over the vertical and diagonal ones in the LH subband (horizontal lowpass filtering followed by vertical highpass filtering).

Since the inter-band dependency is not as useful at lower quadtree levels, we restrict the modeling contexts to the intraband neighbors for significance coding at the pixel quadtree level. It was mentioned that the coding statistics for significance test of the individual child are position-dependent. Hence, significance coding of each child is additionally conditioned on its relative position, $v$, and the significance status of its past coded siblings, $\sigma_{\mathrm{sb}}$.

The look-tables are respectively designed for the subbands of LH and HH orientations. Following the idea of EBCOT, the coefficient matrix from the HL subband is transposed first before the bitplane coding process starts. In this way, the same set of look-up tables can be shared by both the HL and HL subbands. The transposition of the LH subband is avoided in the JPEG 2000 standard by transposing the look-up tables for the LH subband instead.

\section{G. Refinement of significant coefficients}

The same contextual intraband region shown in Fig. 3 is utilized for conditional coding of the refinement of the significant coefficients from LSP. The contextual information is characterized by significance map $\sigma^{\mathrm{p}}(\mathrm{i}, \mathrm{j})$, the significance status with respect to the quantization threshold at the previous bitplane level.

\section{H. Context-dependent de-quantization}

This section presents a new de-quantization algorithm that features a context-dependent strategy for reconstruction of 
subband coefficients. A simple source statistical model is designed for each context with the model parameters estimated from the statistics accumulated at the decoder.

It is known that the optimal representation levels of a scalar quantizer should satisfy the following centroid condition

$$
r_{1}^{*}=\frac{\int_{d_{i}}^{d_{i+1}} x f x(x) d x}{\int_{d_{i}}^{d_{i+1}} f x(x) d x}
$$

Where $r_{i}^{*}$ is the optimal reconstruction value for a given quantization interval $\left[d_{i}, d_{i+1}\right]$ and a probability density function (pdf) $f_{X}(x)$. However, the reconstruction values for the decoded coefficients are typically set to the midpoints of the corresponding quantization intervals in practice either for simplicity of implementation or for lack of knowledge about the source statistics. Such a choice is optimal for the source with uniform probability distribution over the individual decision intervals. For bitplane coding in particular, it is implicitly assumed that the positive and negative coefficients within the dead zone are equally likely and the reconstructed values of all the insignificant pixels are set to zero as a result.

The proposed de-quantization algorithm is based on the two experimental observations:

1) Subband coefficients exhibit strong spatial correlation in both signs and magnitudes

2) The statistics of bitplane samples bear strong resemblances within and across bitplane levels in a given subband and gradually grow into less skew probability distribution from bitplane to bitplane.

Observation (i) has been utilized for context-based arithmetic coding of the subband coefficients in EZBC. Similarly, the coded significant pixels can provide contextual information for reconstruction of their neighboring coefficients. Particularly, the signs of significant pixels are useful for predicting the signs of their neighboring insignificant coefficients which are distributed over the dead zone and are quantized to the zero symbols. Combined with observation (ii), we can estimate the source statistics on a given context using the related probability tables accumulated during the decoding process.

\section{Reconstruction of significant coefficients}

A simple statistical model is adopted for reconstruction of a significant subband coefficient $\left|c_{i}\right| \in\left[x_{0}, x_{0}+\tau_{i}\right)$, as depicted in Fig. 4(a), where $x_{0}$ is the decoded value of $\left|c_{i}\right|$. The quantizer step size, $\tau_{i}$, for $c_{i}$ is given by

$$
\tau_{i}=\left\{\begin{array}{l}
2^{\alpha}, \quad \text { if } c_{i} \text { has been coded during the last pass } n \\
2^{a+1}, \text { otherwise }
\end{array}\right.
$$

The estimated conditional probability

$$
\hat{p}_{0}=\hat{p}_{r}\left\{\left|c_{i}\right|<x_{0}+\frac{\tau_{i}}{2}\left|c_{i}\right| \in\left[x_{0}, x_{0}+\tau_{i}\right] c^{i}\right\}
$$

given a context $c^{i}$ is empirically determined by the related probability tables which were already accumulated during the decoding process. The definition of the contexts is the same as the one used for refinement coding of the significant coefficients. Uniform distribution is then assumed for each half of the quantization interval. The resulting reconstruction level, $\mathrm{r}_{\mathrm{i}}$, is computed by

$$
\begin{gathered}
\tau_{i}=\int_{x_{0}}^{x_{0}+\tau_{i}} x \hat{f}_{x}(x) d x \\
=\frac{2 \hat{p}_{0}}{\tau_{i}} \int_{x_{0}}^{x_{0}+\tau_{i}} x d x+\frac{2\left(1-\hat{p}_{0}\right)}{\tau_{i}} \int_{x_{0}+\tau_{i} / 2}^{x_{0}+\tau_{i}} x d x \\
=x_{0}+\tau_{i}\left(\frac{3}{4}-\frac{1}{2} \hat{p}_{0}\right)=x_{0}+\tau_{i}\left(\frac{3}{4}-\frac{1}{2} \hat{p}_{0}\right)
\end{gathered}
$$

where $\hat{f}_{x}(x)$ represents the estimated conditional probability density function for the magnitude of a significant coefficient $c_{i}$. We note that the reconstruction value $r_{i}$ approaches the midpoint of the quantization bin, the conventional reconstruction value, as the probability distribution becomes less skew $\left(\hat{p}_{O} \rightarrow 1 / 2\right)$

\section{J. Reconstruction of insignificant coefficients}

Our statistical model for reconstruction of insignificant subband coefficients $c \in\left(-\tau_{i}, \tau_{i}\right) \hat{p}_{s p} \rightarrow \frac{1}{2}, r \approx 0$ is depicted in Fig. 4 (b). The quantization threshold $\tau_{i}$ for $\mathrm{c}_{\mathrm{i}}$ is equal to $2^{\mathrm{n}}$ or $2^{n+1}$, depending upon whether $c_{i}$ has been visited during the last bitplane pass. The estimated conditional probabilities

$$
\begin{aligned}
& \hat{p}_{0}=\hat{p}_{r}\left\{\left|c_{i}\right|<\frac{\tau_{i}}{2} \mid c_{i} \in\left(-\tau_{i}, \tau_{i}\right) c^{i}\right\} \text { and } \\
& \hat{p}_{s p}=\hat{p}_{r}\left\{\zeta_{i}=1 \mid c_{i} \in\left(-\tau_{i}, \tau_{i}\right), c^{i}\right\} \text { for }
\end{aligned}
$$
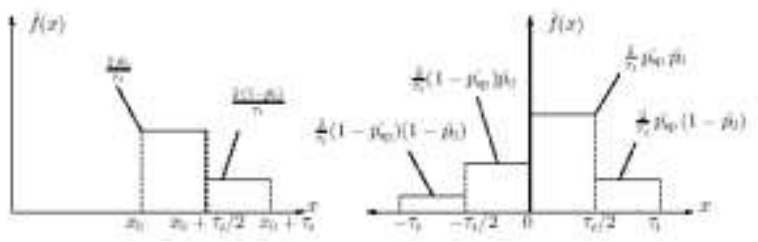

Figure 4 : The assumed conditional probability density function (pdf) for the subband coefficients.

(a) Left: The conditional pdf model for significant

$$
\text { coefficients }\left|c_{i}\right| \in\left[x_{0}, x_{0}+\tau_{i}\right) \text {. }
$$

(b) Right: The conditional pdf model for insignificant

$$
\text { coefficients } c_{i} \in\left(-\tau_{i}, \tau_{i}\right) \text {. }
$$

The right half of the coordinate plane corresponds to $\zeta_{i}=1$ (the sign prediction is correct). The given contexts $\mathrm{C}^{\mathrm{i}}$ are empirically decided by the accumulated probability tables, where $\zeta_{i}$ denotes the correctness of sign prediction for $c_{i}$. 
The definitions of the contexts for estimation of $\hat{p}_{0}$ and $\hat{p}_{s p}$ are the same as the ones employed in significance coding of pixels and sign coding, respectively. Further, we assume the events $\left\{\left|c_{i}\right|<\frac{\tau_{i}}{2} \mid c_{i} \in\left(-\tau_{i}, \tau_{i}\right), c^{i}\right\}$

and

$\left\{\zeta_{i}=1 \mid c_{i} \in\left(-\tau_{i}, \tau_{i}\right) c^{i}\right\}$

are statistically independent so that

$p_{r}\left\{\left|c_{i}\right|<\frac{\tau_{i}}{2} \zeta_{i}=1 \mid c_{i} \in\left(-\tau_{i}, \tau_{i}\right) c^{i}\right\}=$

$p_{r}\left\{\left|c_{i}\right|<\frac{\tau_{i}}{2} \mid c_{i} \in\left(-\tau_{i}, \tau_{i}\right) c^{i}\right\}$

$p_{r}\left\{\left|c_{i}\right|<\frac{\tau_{i}}{2} \mid c_{i} \in\left(-\tau_{i}, \tau_{i}\right) c^{i}\right\}$

\section{Results}

The results at different bpp are shown in Figure 5.

It is shown as the bit rate decreases, the quality of the reconstructed MRI image should degrade. The results are tabulated for two samples as in figure 6 and 7. The PSNR values for different bpp are shown in table 1.

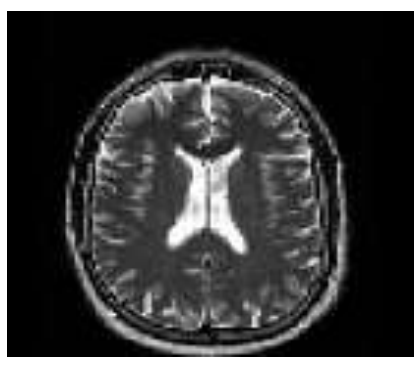

(a) Original MRI image sample1

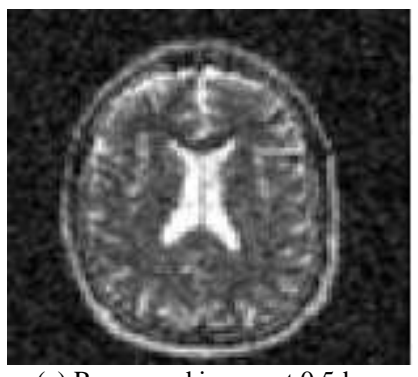

(c) Recovered image at $0.5 \mathrm{bpp}$

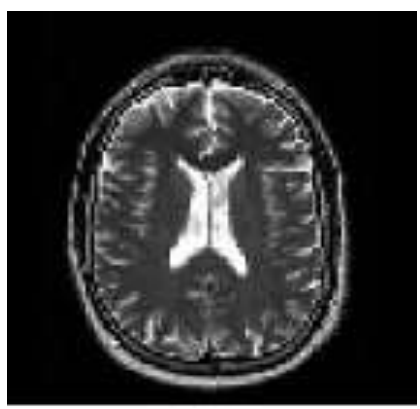

(i)

Original MRI Image sample2

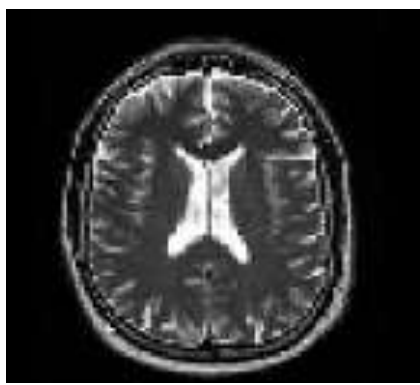

(ii)

Recovered MRI image at 0.9 bpp

Figure 5. Recovered images withdifferent Bits per pixel values.

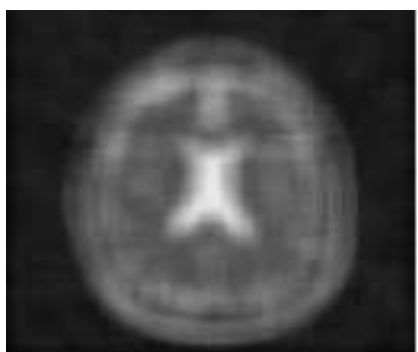

(b) Recovered MRI image sample at $0.1 \mathrm{bpp}$

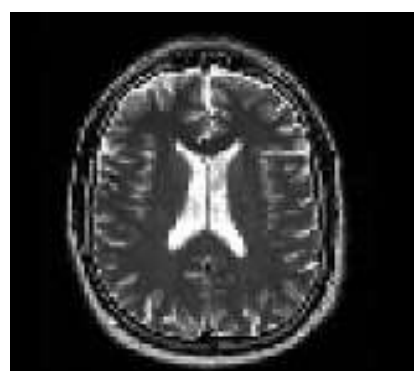

(d) Recovered MRI image sample at $0.9 \mathrm{bpp}$

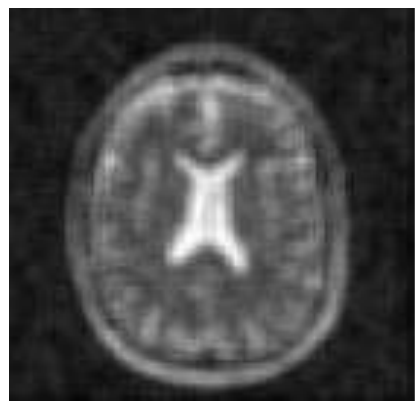

(iii)

Recovered image at $0.1 \mathrm{bpp}$ 


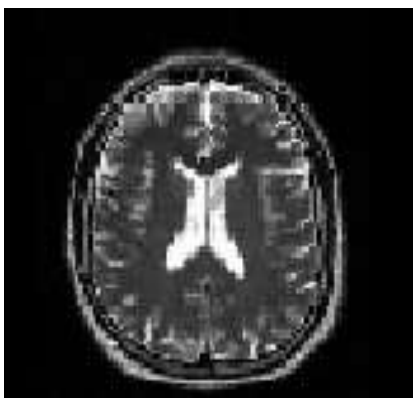

(iv) Recovered MRI image sample at $0.5 \mathrm{bpp}$

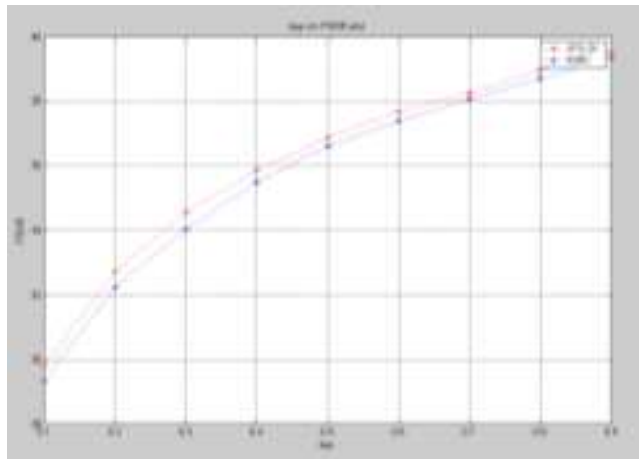

Fig. 6 PSNR v/s bpp plot for the given image

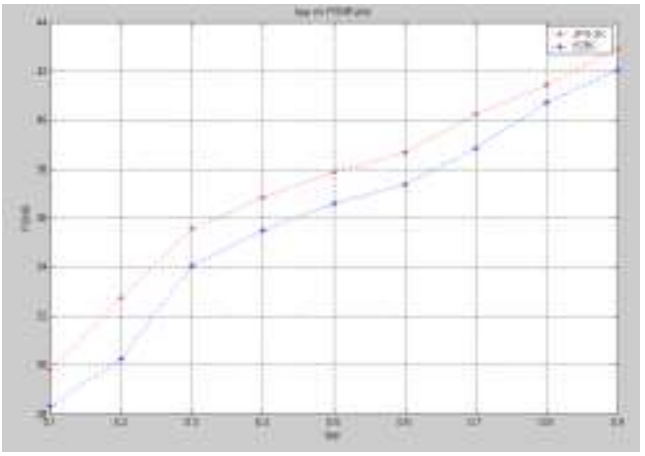

Fig. 7 PSNR v/s bpp plot for the given image

TABLE 1 PSNR V/S BPP PLOT FOR THE GIVEN LEAF IMAGE

\begin{tabular}{|c|c|c|c|c|c|}
\hline \multicolumn{3}{|c|}{ Sample - 1 } & \multicolumn{3}{|c|}{ Sample - 2} \\
\hline bpp & & & bep & & \\
\hline & $J P G-2 K$ & EZBC & & $\mathrm{IP}_{\mathrm{i}-2 \mathrm{~K}}$ & $E Z B C$ \\
\hline 0.1 & 28.7871 & 28.2544 & 0.1 & 29.8107 & 28.3202 \\
\hline 0.2 & 32.4212 & 32.1077 & 0.2 & 32.7202 & 30.2514 \\
\hline 0.3 & 34.5121 & 34.0187 & 0.3 & 35.5479 & 34.0331 \\
\hline 0.4 & 35.5643 & 35.3201 & 0.4 & 36.8422 & 35.4857 \\
\hline 0,5 & 36,7434 & 36.1745 & 0.5 & 37.8623 & 36.5939 \\
\hline 0.6 & 37.7332 & 37.50107 & 0.6 & 38,6650 & 37.3759 \\
\hline 0.7 & 38.3265 & 38.0411 & 0.7 & 40.2581 & 38.8491 \\
\hline 0.8 & 38.8021 & 38.6721 & 0.8 & 41.4390 & 40.7058 \\
\hline 0.9 & 39.6411 & 39.2314 & 0.9 & 42.9218 & 42.0437 \\
\hline
\end{tabular}

\section{CONCLUSION}

This project implements an enhanced image coding system for medical image compression compared to the existing JPEG
2000 system. It is observed that EZW is able to achieve its good performance with a relatively simple algorithm. EZW does not require complicated bit allocation procedures like subband coding does, it does not require training or codebook storage like vector quantization does, and it does not require prior knowledge of the image source like JPEG does (to optimize quantization tables). EZW also has the desirable property, resulting from its successive approximation quantization.

One desirable consequence of an embedded bit stream is that it is very easy to generate coded outputs with the exact desired size. Truncation of the coded output stream does not produce visual artifacts since the truncation only eliminates the least significant refinement bits of coefficients rather than eliminating entire coefficients as is done in subband coding. From the obtained results it is concluded that embedded zero tree wavelet coding takes comparatively less(about $60 \%$ ) time then the JPEG coding system. The coding also shows less percentage of error in medical image compare to the existing JPEG coding system. It is observed that image coded with embedded zerotree wavelet coding shows clearer image than other coding system.

\section{REFERENCES}

[1] A. Said Pearlman, "A new, fast, and efficient image codec based on set partitioning in hierarchical trees,"IEEE Trans. Circuits Syst. Video Technol., vol.6, no.3,1996, pp.243-249.

[2] D.Taubman, "High Performance scalable image compression with EBCOT”, IEEE Trans. Image processing, vol.9, no.7, 2000, pp. $1158-1170$

[3] M.Rabbani and R.Joshi, "An overview of the JPEG 2000 still image compression standard", Signal Processing: Image Communication, vol.17, no.1, 2002, pp.3-48.

[4] S.-T. Hsiang and J.W.Woods, "Embedded image coding using zeroblocks and of subband/wavelet coefficients and context modeling", Proc. IEEE ISCAS'00, vol.3, Geneva Switzerland, may 2000, pp. 662-665.

[5] J.M. Shapiro, "Embedded image coding using zerotrees of wavelet coefficients", IEEE Trans. on signal processing.vol.41, Dec.1993, pp. 3445-3462

[6] G.K. Wallace, "The JPEG still picture compression standard", Communications of the ACM, vol. 34, April 1991, pp.30-44.

[7] W.P. Pennebaker and J.L. Mitchell. JPEG Still Image Data Compression Standard. New York: Van Nostrand Reinhold

[8] J.Woods. ed., Subband Image Coding. Kluwer Academic Publishers. 1991.

[9] M.Vetterli and J. Kovacevic, Wavelets and Subband Coding. Englewood Cliffs.NJ: Prentice-Hall, 1995.

[10] M. Antonini.M. Barlaud, P. Mathieu, and I. Daubechies, "Image coding using wavelet transform “, IEE Trans. Image Processing, vol. 1, pp. 205220, 1992

[11] S.-T. Hsiang and J. W. Woods, "Highly scalable and perceptually tuned embedded subband/wavelet image coding,"in SPIE Conference on VisualCommunications and Image Processing, vol. 4671, (San Jose, CA), Jan. 2002, pp. 1153-1164.

[12] N. S. Jayant and P. Noll, Digital Coding of Waveforms. Englewood Cli.s, NJ: Prentice-Hall, 1984.

[13] A. K. Jain, Fundamentals of Digital Image Processing. Englewood Cli.s, NJ: Prentice-Hall, 1989.

[14] S. Rane, A. Aaron, and B. Girod, "Systematic Lossy Forward Error Protection for Error Resilient Digital Video Broadcasting," in Proc. VCIP 2004, San Jose, CA, USA. 
[15] A. Sehgal, A. Jagmohan, and N. Ahuja, "Wyner-Ziv coding of video: An error-resilient compression framework," IEEE Trans. Multi., vol. 6, no. 2, Apr. 2004, pp. 249-258.

[16] C. Tillier and B. Pesquet-Popescu, "3D, 3-Band, 3-Tap Temporal Lifting For Scalable Video Coding," in Proc. ICIP 2003, Barcelona, Spain, 2003,Sept. 14-17.

[17] C. Tillier, B. Pesquet-Popescu, and M. Van der Schaar, "Multiple Description Scalable Video Coding," in Proc. EUSIPCO 2004, Vienna, Austria, 2004,Sept. 6-10.

[18] C. Tillier and B. Pesquet-Popescu, "A New 3-band MCTF Scheme For Scalable Video Coding," in Proc. PCS 2004, San Francisco, CA, USA, 2004, Dec. 15-17.

[19] C. Brites, J. Ascenso and F. Pereira, "Improving Transform Domain Wyner-Ziv Video Coding Performance," in Proc. ICASSP 2006, Toulouse, France, 2006, May 14-19.

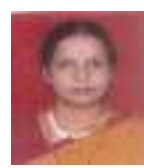

\section{AUTHORS PROFILE}

LalithaY.S was born on December 7, 1969 in India. She received B.E degree in Electronics and Communication Engineering and M.E. degree in Power Electronics from Gulbarga University Gulbarga, India, in 1991 and 2002 respectively. She is working as Professor in Appa Institute of Engineering \& Technology, Gulbarga, India. Her research interests include image Processing, Wavelet Transform coding.

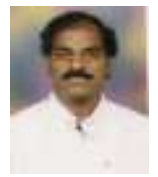

Mrityunjaya V Latte was born on April $25^{\text {th }} 1964$ in India. He received B.E. degree in electrical Engineering and M.E. degree in Digital electronics from S.D.M. College of Engineering \& Technology, Dharwad, India in 1986 \& 1995 respectively. He was awarded Ph.D. degree in 2004 for his work in the area of digital signal processing. He is working as Principal, JSS Academy of Technical Education, Bangalore, India. His research interests include coding, image processing and multiresolution transforms. He received a best paper award for his paper in a national conference NCSSS -2002 held at Coimbatore, India. 